\title{
Avaliação de colesterol e triglicerídeos séricos em cães saudáveis suplementados com ômega n-3
}

\author{
[Evaluation of serum cholesterol and triglycerides in healthy dogs \\ supplemented with ômega-3 $N$ ] \\ M.M.G. Araujo, T.H.Y. Santos, M.L.G. Lourenço*, R.K. Takahira, \\ L.H.A. Machado, L.R. Carvalho \\ ${ }^{1}$ Faculdade de Medicina Veterinária e Zootecnia - UNESP - Botucatu, SP \\ ${ }^{2}$ Instituto de Biociências - UNESP - Botucatu, SP
}

\begin{abstract}
RESUMO
A análise da concentração sérica de colesterol e triglicerídeos foi realizada em 20 cães, sem raça definida, saudáveis, 10 machos e 10 fêmeas, previamente e após a suplementação por 30 dias com ácidos graxos poli-insaturados de cadeia longa derivados do ômega n-3 (497mg ácido docosa-hexaenoico e 780mg ácido eicosapentanoico). A concentração sérica de colesterol apresentou redução significativa após a suplementação em ambos os sexos $(271,6 \pm 79,8 \mathrm{mg} / \mathrm{dL} ; 236,2 \pm 67,6 \mathrm{mg} / \mathrm{dL}$, antes e após suplementação, respectivamente). Em relação à concentração sérica de triglicerídeos, houve redução apenas nas fêmeas $(57,8 \pm 12,1 \mathrm{mg} / \mathrm{dL} ; 45,2 \pm 7,8 \mathrm{mg} / \mathrm{dL}$, antes e após suplementação, respectivamente), não havendo efeito da suplementação nos machos.
\end{abstract}

Palavras-chave: cão, ômega n-3, colesterol, triglicerídeos

\begin{abstract}
The analysis of serum cholesterol and triglycerides was performed in 20 healthy mongrel dogs, 10 male and 10 female, before and after supplementation for 30 days with fatty acids of long chain polyunsaturated derived from omega-3 n (497mg docosahexaenoic acid and 780mg eicosapentaenoic acid). The serum cholesterol presented a significant reduction after supplementation in both sexes (271.6 $\pm 79.8 \mathrm{mg} / \mathrm{dL} ; 236,2 \pm 67,6 \mathrm{mg} / \mathrm{dL}$, before and after supplementation respectively). Regarding serum triglycerides, there was a reduction only in females $(57.8 \pm 12,1 \mathrm{mg} / \mathrm{dL} ; 45.2 \pm 7,8 \mathrm{mg} / \mathrm{dL}$, before and after supplementation respectively), with no effect of supplementation in males.
\end{abstract}

Keywords: dog, omega n-3, cholesterol, triglycerides.

\section{INTRODUÇÃO}

Os efeitos da hiperlipidemia persistente em cães não estão totalmente esclarecidos. Em comparação aos seres humanos, os gatos e os cães são tipicamente resistentes à doença arterial coronariana, ao infarto do miocárdio, a acidente vascular cerebral e à aterosclerose (Bauer, 2007). Cães e a maioria dos mamíferos são considerados mamíferos HDL - lipoproteína de alta densidade -, pois apresentam predominância dessa lipoproteína na circulação, diferentemente

Recebido em 18 de julho de 2011

Aceito em 6 de agosto de 2012

E-mail: mege@uol.com.br dos humanos, que são classificados como mamíferos LDL - lipoproteína de baixa densidade -, mais sensíveis à elevação do colesterol e desenvolvimento de aterosclerose (Schenck, 2006). O desenvolvimento de aterosclerose em cães está associado à ocorrência de hiperlipidemia secundária ao hipotireoidismo, diabetes mellitus e pancreatite (Schenck, 2006).

De modo geral, recomenda-se o tratamento da hiperlipidemia quando a concentração sérica de triglicerídeos for superior a $500 \mathrm{mg} / \mathrm{dL}$ ou de colesterol acima de 500mg/dL (Xenoulis, 
2010). A terapia envolve a instituição de dieta com baixos teores de gordura $(<20 \%$ de gordura por energia metabolizável) por seis a oito semanas, bem como a utilização de suplementação com óleo de peixe $(1 \mathrm{~g} / 4,55 \mathrm{~kg}$ peso corpóreo) rico em ômega 3 (Elliot e Schenck, 2010).

Ácidos graxos são constituídos por uma cadeia longa de hidrocarbonetos com um número par de átomos de carbono. São denominados baseandose no número de átomos - do quarto ao $26^{\circ}-$, no número de duplas ligações $\left(\begin{array}{lllll}0 & \text { a } & 6\end{array}\right)$ e na localização das duplas ligações - no terceiro, sexto, sétimo ou nono carbono. A maioria dos ácidos tem a denominação química C18:1 n-9 ácido graxo com 18 carbonos, uma dupla ligação que está localizada no nono carbono -, bem como um nome em comum: ácido oleico (Leaf et al., 2005).

Existe uma série de ácidos graxos ômega 3, contudo os mais comuns são o ácido linolênico (ALA), o ácido eicosapentaenoico (EPA) e o ácido docosa-hexaenoico (DHA) (Leaf et al., 2005; Freeman, 2010). Os ácidos graxos insaturados, denominados ômega 3, vêm sendo cada vez mais utilizados na dieta de animais devido aos seus inúmeros efeitos benéficos ao organismo. Os benefícios do ômega $3 \mathrm{em}$ humanos são bem descritos já há alguns anos, e o consumo de peixe ou de óleo de peixe é recomendado para humanos (Freeman, 2010).

Entre os benefícios do ômega 3, destacam-se: prevenção da isquemia ventricular fatal induzida por arritmias em animais (Mclennan, 1993; Yang, 1993; Billman et al., 1999); proteção renal (Brown, 1998); diminuição satisfatória no controle do prurido em cães atópicos (Scott, 1988; Miller, 1989; Paradis, 1991); modulação da expressão de proteínas no tecido adiposo, podendo atuar como reguladores transcricionais de alguns genes relacionados ao metabolismo de lipídios (Queiroz, 2009); diminuição da concentração de trigliceróis (Etherton, 2002), devido à redução da lipogênese hepática ocasionada pela inibição de duas enzimas envolvidas na síntese desse lipídio pelo fígado (Harris, 2006); e redução da pressão arterial e do processo inflamatório (Din, 2004).

Alguns dos benefícios da suplementação com os ácidos graxos poli-insaturados de cadeia longa derivados do ômega 3 são seu efeito positivo na redução dos níveis de lipídios séricos de triacilglicerois e de lipoproteínas de muito baixa (VLDL) densidade; a diminuição do colesterol e de lipoproteína de baixa densidade (LDL); e a elevação de lipoproteína de alta densidade (HDL), responsável pelo transporte de colesterol ao fígado para ser metabolizado e eliminado, impedindo a deposição dele nas paredes das artérias (Serrano, 2002).

A diminuição dos níveis séricos de triglicerídeos e colesterol com a suplementação com ômega 3 ocorre pela diminuição das concentrações séricas de apoproteína CIII, a qual inibe a atividade da lipoproteína lipase e, consequentemente, favorece a depuração de triglicerídeos sanguíneos. Os PUFAS derivados do ômega 3 regulam a expressão gênica da apoproteína CIII, o que promove a inibição da síntese hepática de lipoproteínas carreadoras de triglicerídeos como VLDL, diminui a absorção intestinal de lipídios e aumenta a secreção de colesterol pela bile (Mullick et al., 2002; Adkins, 2010).

Segundo Etherton (2002), o consumo de peixe ou a ingestão de cápsulas de óleo de peixe contendo PUFA n-3 pode causar alterações no perfil lipídico sérico. Não foram descritos estudos que avaliaram a segurança da suplementação com PUFAS derivados do ômega 3 - óleo de peixe a longo prazo em cães, contudo em outras espécies, como no homem, a adição de óleo de peixe à dieta não demonstra inocuidade (Le Blanc et al., 2005).

De acordo com Le Blanc et al. (2005), ao avaliarem os efeitos da suplementação da dieta de cães com óleo de peixe nos lipídios sanguíneos, não houve diferença significativa na concentração sérica de colesterol após seis a 12 semanas de suplementação; por outro lado, a concentração de triglicerídeos diminuiu significativamente após 12 semanas, quando comparada à dos cães pertencentes ao grupocontrole, não suplementados.

Embora os cães sejam raramente estudados em relação a distúrbios relacionados à hiperlipidemia primária, são especialmente úteis na avaliação de compostos e medicamentos para humanos. Desse modo, o conhecimento da concentração sérica de colesterol e triglicerídeos, 
bem como do metabolismo de lipoproteínas, é importante para fins comparativos. O intuito do estudo das respostas metabólicas lipídicas mediante a suplementação com PUFAS derivados do ômega 3 tem sido o de compreender os efeitos da modificação dietética em animais clinicamente saudáveis. $\mathrm{O}$ conhecimento gerado, dessa forma, permite avaliar os benefícios dietéticos em animais saudáveis. Além disso, possibilita melhor interpretação das interações da dieta na doença (Bauer, 2007).

Mediante o exposto, o objetivo do presente estudo foi avaliar a concentração sérica de colesterol e triglicerídeos em cães sem raça definida, saudáveis, suplementados por 30 dias com PUFAS derivados do ômega 3.

\section{MATERIAL E MÉTODOS}

Foram utilizados 20 cães adultos, sem raça definida e clinicamente saudáveis, sendo 10 machos, com média de peso de $10,2 \pm 22,3 \mathrm{~kg}$, e 10 fêmeas, com média de peso de $7,9 \pm 18,1 \mathrm{~kg}$. Entre as fêmeas, três eram inteiras e sete ováriossalpingo-histerctomizadas. Os animais, imunizados com vacina polivalente contra cinomose, leptospirose, hepatite infecciosa e parvovirose, foram mantidos com ração comercial $(21 \%$ de proteína bruta, $12 \%$ de umidade, $5 \%$ de extrato etéreo, $6,5 \%$ de matéria fibrosa com valor nutricional de $3300 \mathrm{kcal} / \mathrm{kg}$ de alimento) fornecida uma vez ao dia e água $a d$ libitum.

Após pesagem dos cães e avaliação clínica (exame físico), determinaram-se as concentrações séricas de colesterol e triglicerídeos previamente à suplementação dietética com PUFAS e após a suplementação por 30 dias, por via oral, com duas cápsulas de $2 \mathrm{~g}$ de ômega 3 (Fórmula Médica. Farmacêutica responsável: Cintia Graziela Squissato Basqueira, CRF-SP 27.191, Av. Moema, 678, Moema, São Paulo), contendo por unidade $221,83 \mathrm{mg}$ de EPA e $136,17 \mathrm{mg}$ de DHA, por animal. A dose foi estabelecida com base em estudos prévios com ômega 3 em cães (Freeman et al., 1998; Smith et al., 2007). Os cães receberam a suplementação com ômega 3 diariamente, pela manhã.
Os animais foram submetidos a jejum alimentar por um período superior a 12 horas, e as amostras de sangue para realização do perfil lipídico - colesterol e triglicerídeos - foram colhidas por meio de punção da veia jugular, com agulha hipodérmica e seringas plásticas descartáveis, em dois momentos - antes e após a suplementação por 30 dias. Após a separação do soro por centrifugação a 3000 rotações por minuto durante oito minutos, imediatamente após a colheita do sangue total, a amostra de soro foi estocada em tubos de polietileno, congelados imediatamente e armazenados a $-80^{\circ} \mathrm{C}$, até o momento do processamento das amostras.

O soro foi descongelado em temperatura ambiente no momento da realização do teste e processado imediatamente. A análise de colesterol total (Kit CHOL - Ortho-Clinical Diagnostics Johnson \& Johnson - Vitros ${ }^{\circledR}$ Chemistry Products - UK) e de triglicerídeos (kit TRIG- Ortho-Clinical Diagnostics Johnson \& Johnson - Vitros ${ }^{\circledR}$ Chemistry Products - UK) foi realizada pelo método enzimático colorimétrico, com a utilização de kits comerciais em aparelho automático (Cobas Mira $\mathrm{S}^{\circledR}$ - Roche - Germany). Os valores foram expressos a $37^{\circ}$, em $\mathrm{mg} / \mathrm{dL}$. No presente estudo, utilizaram-se os valores de referência para cão adulto, segundo Tilley (2007), de 112 a 328mg/dL para colesterol e 20 a $150 \mathrm{mg} / \mathrm{dL}$ para triglicerídeos.

Após a obtenção dos dados, como o intuito de comparar os momentos - antes e após a suplementação - e os grupos - machos e fêmeas -, para as variáveis que apresentaram distribuição normal e homogeneidade de variâncias, foi utilizada a análise de perfil, com significância de $5 \%$.

O presente estudo foi aprovado pelo Comitê de Ética no Uso de Animais da CEUA-FMVZ, Botucatu, sob o protocolo 003/10.

\section{RESULTADOS E DISCUSSÃO}

Observou-se diferença significativa $(\mathrm{P}<0,001)$ na concentração sérica de colesterol após a suplementação com PUFAS derivados de ômega 3 (Tab. 1), tanto em machos como em fêmeas. Os valores de colesterol obtidos antes e após a suplementação encontravam-se dentro das concentrações de referência consideradas para cães por Tilley (2007). Estes resultados foram 
compatíveis com os citados por Schenk (2006), em que dietas contendo EPA e DHA induziram decréscimo na concentração sérica de colesterol em ratos, pintos, cães com insuficiência renal, coelhos e gatos, e diferiram dos descritos por Le Blanc et al. (2005), em que a suplementação não exerceu efeitos sobre a concentração de colesterol.

O óleo de peixe marinho possui efeito benéfico na hiperlipidemia por estimulação na atividade da lipoproteína lipase, diminuindo a absorção intestinal de glicose e lipídios e aumentando a secreção de colesterol pela bile (Levy et al., 1993; Adkins, 2010).

A lipoproteína lipase arterial (LpL) tem sido correlacionada com o acúmulo de colesterol na parede arterial em ratos, modelos para aterosclerose (Corey, 1977). Ao contrário dos ácidos graxos saturados, o ômega 3 tem efeitos diferentes no $\mathrm{LpL}$ arterial e na deposição de colesterol. Em humanos, estudos in vitro demonstraram que os ácidos graxos n-3 diminuíram a secreção de $\mathrm{LpL}$ e induziram a apoptose de macrófagos e células do músculo liso, inibindo a aterogênese (Chinetti, 1998). Os ácidos graxos n-3, como o EPA, podem inibir a atividade da LpL em 50-60\% (Chang, 2009). Os mecanismos por trás da redistribuição de $\mathrm{LpL}$ arterial ainda não estão bem esclarecidos em animais.

Segundo Simão (2010), em humanos a ingestão de cápsulas de óleo de peixe diminuiu a concentração de triglicerídeos, porém, ao contrário do presente estudo, a concentração de colesterol total aumentou.

Tabela 1. Concentração sérica de colesterol em cães sadios antes e após a suplementação com PUFAS derivados do ômega 3

\begin{tabular}{ccc}
\hline Sexo & Antes & Após \\
\hline Fêmea & $288,0 \pm 93,4$ & $252,1 \pm 74,7$ \\
Macho & $255,1 \pm 64,3$ & $220,2 \pm 59,3$ \\
Média geral & $271,6 \pm 79,8 \mathrm{a}$ & $236,2 \pm 67,6 \mathrm{~b}$ \\
\hline
\end{tabular}

Letras distintas nas linhas indicam diferença entre momentos $(\mathrm{P}<0,001)$

A concentração sérica de triglicerídeos apresentou diferença entre os momentos apenas nas fêmeas, sendo a média mais alta previamente à suplementação com PUFAS derivados de ômega 3 (Tab. 2). Ocorreu diminuição da concentração após 30 dias, demonstrando interação de sexo versus o momento $(\mathrm{P}=0,03)$. Houve diferença entre machos e fêmeas previamente à suplementação, sendo a concentração sérica de triglicerídeos superior nas fêmeas. Todos os valores descritos neste estudo assemelham-se aos obtidos por Tilley (2007). A concentração sérica de triglicerídeos em machos foi mais baixa que a das fêmeas e possivelmente por esse motivo não sofreu influência da suplementação com ômega 3. Talvez em machos com hipertrigliceridemia, a suplementação promovesse a diminuição da concentração sérica. A diferença entre fêmeas e machos quanto à concentração de triglicerídeos não foi descrita anteriormente em cães, embora em humanos a diferença entre sexos tenha sido mencionada em um estudo que avaliou a ingestão de ácidos graxos e a concentração sérica devido à ingestão em proporções diferentes entre homens e mulheres (Christensen et al., 1999).

Tabela 2. Concentração sérica de triglicerídeos em cães sadios antes e após a suplementação com PUFAS derivados do ômega 3

\begin{tabular}{ccc}
\hline Sexo & Antes & Após \\
\hline Fêmea & $57,8 \pm 12,1 \mathrm{aA}$ & $45,2 \pm 7,8 \mathrm{bA}$ \\
Macho & $46,4 \pm 9,8 \mathrm{aB}$ & $46,4 \pm 11,1 \mathrm{aA}$ \\
\hline
\end{tabular}

Letras distintas minúsculas na linha e maiúsculas na coluna indicam diferenças entre momentos e entre $\operatorname{sexos}(\mathrm{P}=0,03)$.

Diferentemente do descrito por Le Blanc et al. (2005), em que a suplementação com óleo de peixe com PUFAS derivados do ômega $3 \mathrm{em}$ cães saudáveis levou à redução significativa da concentração plasmática de triglicerídeos, no presente estudo a diminuição ocorreu apenas em fêmeas. Essa diferença pode estar relacionada à ausência de esteroides estrogênicos, pois este estudo utilizou sete fêmeas ovariossalpingohisterectomizadas. Segundo Schmidt et al. (2004), a concentração de triglicerídeos em fêmeas após a histerectomia tende a elevar-se. A ausência do efeito de sexo sobre a concentração de colesterol pode ser explicada pelo fato de as fêmeas, após a ovários-salpingo-histerectomia, perderem a vantagem do efeito estrogênico protetor, que se reflete na elevação de LDL, diminuição de HDL e consequente elevação do colesterol sérico, equiparando-se aos machos, que tendem a apresentar LDL e colesterol mais elevados durante toda a vida (Joosens, 1988; Schimidt et al., 2004). 


\section{CONCLUSÕES}

A suplementação dietética de animais sadios com PUFAS derivados do ômega 3 tem influência significativa ao promover a diminuição da concentração de colesterol tanto em machos como em fêmeas. Em relação aos triglicerídeos, pode-se concluir que há diferença entre machos e fêmeas, e que a suplementação diminui a concentração sérica somente nas fêmeas. Embora a suplementação com PUFAS derivados do ômega 3 seja amplamente indicada pela literatura no tratamento das hiperlipidemias em cães, estudos sobre os efeitos de tal suplementação no metabolismo lipídico em cães saudáveis são escassos.

\section{REFERÊNCIAS}

ADKINS, W.; KELLY, D.S. Mechanisms underlying the cardioprotect effects of omega-3 polyunsatured fatty acids. J. Nutr. Biol., v.21, p.781-792, 2010.

BAUER, J.E. Responses of dogs to dietary omega-3 fatty acids. J. Am. Vet. Med. Assoc., v.231, p.1657-1661, 2007.

BILLMAN, G.E.; KANK, J.X.; LEAF, A. Prevention of sudden cardiac death by dietary pure omega-3 polyunsaturated fatty acids in dogs. Circulation, v.99, p.2452-2457, 1999.

BROWN, S.A.; BROWN, C.A.; CROWELL, W.A. et al. Beneficial effects of chronic administration of dietary $\omega-3$ polyunsaturated fatty acids in dogs with renal insufficiency. $J$. Lab. Clin. Med., v.131, p.447-455, 1998.

CHANG, C.L.; SEO, T.; MATSUZAKI, M. et al. N-3 Fatty Acids Reduce Arterial LdlCholesterol Delivery and Arterial Lipoprotein Lipase Levels And Lipase Distribution. Arterioscler. Thromb. Vasc. Biol., v.29, p.555$561,2009$.

CHINETTI, G.; GRIGLIO, S.; ANTONUCCI, M. et al. Activation of proliferator-activated receptors alpha and gamma induces apoptosis of human monocyte-derived macrophages. J. Biol. Chem., v.273, p.25573-25580, 1998.

CHRISTENSEN, J.H.; CHRISTENSEN, M.S.; DYERBERG, J.; SCHMIDT, E.B. Heart rate variability and fatty acid content of blood cell membranes: a dose-response study with $\mathrm{n}-3$ fatty acids. Am. J. Clin. Nutr., v.70, p.331-337, 1999.
COREY, J.E.; ZILVERSMIT, D.B. Effect of cholesterol feeding on arterial lipolytic activity in the rabbit. Atheros., v.27, p.201-212, 1977.

DIN, J.N.; NEWBY, D.E.; FLAPAN, A.D. Omega 3 fatty acids and cardiovascular disease fishing for a natural treatmente. Br. Med. J., v.328, p.30-35, 2004.

ELLIOT, D.A.; SCHENCK, P.A. Dietary and medical consideration in hyperlipidemia. In: ETTINGER, S.J.; FELDMAN, E.C. Textbook of veterinary internal medicine. St. Louis: Elsevier, 2010. p.710-715.

ETHERTON, P.M.K.; HARRIS, W.S.; APPEL, L.J. Nutrition Committee. Fish consumption, fish oil, omega-3 fatty acids, and cardiovascular disease. Circulation, v.106, p.47-57, 2002.

FREEMAN, L.M. Beneficial effects of omega-3 fatty acids in cardiovascular disease. J. Small Anim. Pract., v.51, p.462-470, 2010.

FREEMAN, L. M., RUSH, J. E., KEHAYIAS, J. J. et al. Nutritional alterations and the effect of fish oil supplementation in dogs with heart failure. J. Vet. Int. Med., v.12, p. 440-448, 1998.

HARRIS, W.S.; BULCHANDANI, D. Why do omega-3 fatty acids lower serum triglycerides? Curr. Opin. Lipidol., v.17, p.387-393, 2006.

JOOSENS, J.V. Mechanisms of hypercholesterolemia and atherosclerosis. Acta. Cardiol., v.29, p.63-83, 1988.

LEAF, A.; XIAO, Y.F.; KANG, J.X.; BILLMAN, G.E. Membrana effects of the n-3 fish oil fatty acids, with prevent fatal ventricular arrhtythmias. J. Membrane. Biol., v.206, p.129139, 2005.

LE BLANC, C.J.; BAUER, J.E.; HOOSGOOD, G.; MAULDIN, G.E. Effect of dietary fish oil and vitamin $\mathrm{E}$ supplementation on hematologic and serum biochemical analytes and oxidative status in young dogs. Vet. Ther., v.6, p.325-340, 2005.

LEVY, E.; THIBAULT, L.; TURGEON, J. Beneficial effects of fish oil supplements on lipids, lipoproteins, and lipoprotein lipase in patients with glycogen storage disease type I. Am. J. Clin. Nutr., v.57, p.922-929, 1993. 
MCLENNAN, P.L. Relative effects of dietary saturated, monounsaturated, and polyunsaturated fatty acids on cardiac arrhythmias in rats. Am. J. Clin. Nutr., v.57, p.207-212, 1993.

MILLER JR, W.H.; GRIFFIN, C.E.; SCOTT, D.W.; ANGARANO, D.K. et al. Clinical trial of DVM Derm Caps in the treatment of allergic diseases in dogs: A nonblinded study. J. Am. Anim. Hosp. Assoc., v.25, p.163-168, 1989.

MULLICK, A.E.; DECKELBAUM, R.J.; GOLDBERG, I.J.; AL-HAIDERI, M. et al. Apolipoprotein $\mathrm{E}$ and lipoprotein lipase increase triglyceride-rich particle binding but decrease particle penetration in arterial wall. Arterioscler. Thromb. Vasc. Biol., v.22, p.2080-2085, 2002.

PARADIS, M.; LEMAY, S.; SCOTT, D.W. The efficacy of clemastine (Tavist), a fatty acidcontaining product (Derm Caps), and the combination of both products in the management of canine pruritus. Vet. Dermatol., v.2, p.17-20, 1991.

QUEIROZ，J.C.F.; ALONSO-VALE， M.I.C.; CURI, R.; LIMA, F.B. Controle da adipogênese por ácidos graxos. Arq. Bras. Endocrinol. Metab., v.53, p.582-594, 2009.

SCHENCK, P.A. Canine hyperlipidemia: causes and nutritional management. In: PIBOT, P,; BIOURGE, V.; ELLIOT, D.A. Encyclopedia of canine clinical nutrition. Paris: Aniwa SAS, 2006. p.222-251

SCHMIDT, C.; LOPES, M.D.; SILVA, M.C.; FIGHERA, R.A. et al. Perfil lipoproteico de cadelas submetidas a ovário-histerectomia com e sem reposição estrogênica. Arq. Bras. Med. Vet. Zootec., v.56, p.449-456, 2004.
SERRANO, P.P. Desempenho, parâmetros sanguíneos, perfil graxo e conteúdo de colesterol na carcaça de frango de corte alimentados com diferentes fontes de ácidos graxos. 2002. 64f. Dissertação (Mestrado em Zootecnia) Faculdade de Ciências Agrárias e Veterinárias, Universidade Estadual Paulista, Jaboticabal, SP.

SCOTT, D.W.; BUERGER, R.G. Nonsteroidal anti-inflammatory agents in the management of canine pruritus. J. Am. Anim. Hosp. Assoc., v.24, p.425-428, 1988.

SIMÃO, A.N.C.; GODENY, P.; LOZOVOY, M.A.B.; DICHI, J.B. et al. Efeito dos ácidos graxos n-3 no perfil glicêmico e lipídico, no estresse oxidativo e na capacidade antioxidante total em pacientes com síndrome metabólica. Arq. Bras. Endocrinol. Metab., v.54, p.54-55, 2010.

SMITH, C.E.; FREEMAN, L.M.; RUSH, J.E.; CUNNINGHAM, S.M. et al. Omega-3 fatty acids in boxer dogs with arrhythmogenic right ventricular cardiomyopathy. J. Vet. Intern. Med., v.21, p.265-273, 2007.

TILLEY, P.L.; SMITH JR, F.W.K. The five Minute Veterinary Consult: Canine and Feline. 4nd ed. Iowa: Blackwell Publishing Professional, 2007. 1578p.

YANG, B.; SALDEEN, T.G.P.; NICHOLS, W.N.; MEHTA, J.L. Dietary fish oil supplementation attenuates myocardial dysfunction and injury caused by global ischemia and reperfusion in isolated rat hearts. J. Nutr., v.12, p.2067-20741, 1993.

XENOULIS, P.G.; STEINER, J.M. Lipid metabolism and hyperlipidemia in dogs. Vet. J., v.183, p.12-21, 2010. 